\title{
Arduino Nano Tabanlı Bir Eğitim Robotu Geliştirilmesi: myNanoBot Araștırma Makalesi/Research Article
}

\author{
Erkan DURAN \\ Ovaköy Ortaokulu, Altıeylül, Balıkesir, Türkiye \\ duran.erkan@gmail.com \\ (Geliş/Received:06.06.2021; Kabul/Accepted:05.11.2021) \\ DOI: $10.17671 /$ gazibtd. 948478
}

\begin{abstract}
Özet- Bu çalışmanın amac1, temel kodlama ve robotik eğitimlerinde genellikle tercih edilen hazır robot kitlerine alternatif bir eğitim robotu geliştirmektir. Bu amaçla hem öğretmenler hem de öğrenciler tarafindan temin edilmesi ve montajı kolay; açık kaynak kodlu Arduino mikrodenetleyicisi ile çeşitli aktüatör ve sensörlere sahip düşük maliyetli bir eğitim robotu geliștirilmiștir. İlgili robot, yine araștırmacı tarafindan yazılan uzantı ile mBlock yazılımı (3.4.12 sürümünde) blok tabanlı olarak kolayca programlanabilmektedir. Bununla birlikte robot, yapılan uygulamaları herhangi bir derleme ve yükleme işlemine gerek kalmaksızın mBlock yazılımı Scratch modunda USB ve bluetooth bağlantıs1 üzerinden eş zamanlı bir șekilde çalsştırabilmektedir. Bu amaçla Makeblock mBot seri port protokolü ile Makeblock elektronik modüllerine ait aygıt yazılımları robota uyarlanmıs ve robotun kendi aygıt yazılımı oluşturulmuştur. Robotun geliştirilmesinde Arduino Nano geliştirme kartı, bluetooth modülü, ultrasonik mesafe sensörü, ışık sensörü, ses sensörü, RGB LED, buzzer, motor sürücü kartı ve DC motor kullanılmıştır. Arduino Uno ve Arduino Mega geliştirme kartları ile de test edilen robot, temel kodlama ve robotik eğitimlerinde kullanılabilir ve farklı sensörler eklenerek geliştirilebilir.
\end{abstract}

\section{The Development of an Arduino Nano Based Educational Robot: myNanoBot}

\begin{abstract}
The aim of this study is to develop an alternative educational robot to generally preferred ready robot kits in basic coding and robotic educations. For this purpose, easy to supply and assemble by both teachers and students, a low-cost educational robot equipped with various sensors and actuators was developed using open source Arduino microcontroller. And again with written extension by the researcher, the robot can be easily programmed as block-based in mBlock software (version 3.4.12). At time same time, the robot can run applications simultaneously without the need for compilation and upload in mBlock software Scratch mode via USB and bluetooth connection. For this purpose, Makeblock mBot serial port protocol and firmwares of Makeblock electronic modules were adapted to the robot and the robot's own firmware were created. In the development of the robot, Arduino Nano development card, bluetooth module, ultrasonic distance sensor, light sensor, sound sensor, RGB LED, buzzer, motor driver card and DC motor were used. Tested also with Arduino Uno and Arduino Mega development cards, the robot can be used in basic coding and robotic educations and can be developed by adding different sensors.
\end{abstract}

Keywords - Arduino, educational robot, mBlock, extension, Scratch mode 


\section{GIRIŞ (INTRODUCTION)}

Kodlama, çağımızın bilişim teknolojilerini yönlendiren alanların temeli olarak görülmekte ve "geleceğin dili", "yeni çağın alfabesi” olarak nitelendirilmektedir [1]. Programlama öğretimi ile ilgili yapılan çalışmalar değerlendirildiğinde bütün dünyada öğrencilerin programlamayı öğrenme konusunda çeşitli sıkıntılar yaşadıkları belirtilmektedir [2]. Bununla birlikte programlama dillerinin küçük çocuklar tarafindan bile kullanımını kolaylaştıran blok tabanlı programlama dilleri olarak bilinen yeni görsel programlama dilleri ile öğrenciler, programlama temellerini ve becerilerini kolayca öğrenebilmektedir [3]. Bu dillerden biri de MIT Medya Laboratuvarında geliştirilen sürükle-bırak yöntemi ile çalışan ve oldukça geniş kullanıma sahip olan Scratch programıdır [4]. Eğitimlerde Scratch yazılımının kullanımı, programlama öğretimini soyut ve karmaşık yapıdan çıkararak somutlaştırmakta, öğrencilerin ilgi ve motivasyonlarını da yükseltmektedir [5].

Son zamanlarda popülerliği artan eğitim robotları ise, kodlamanın sonuçlarını gerçek dünyada görmeyi sağlayan, soyut kavramları somutlaştırarak öğrenmeyi kolaylaştıran öğretim materyalleridir [6]. mBot, Lego, Makey Makey, VEX en sık karşılaşılan hazır robotik eğitim kitleri arasında yer almaktadır.

Arduino ise düşük maliyetli, açık kaynak kodlu, kolay programlanabilir yapıda, kullanımı için daha az teknik bilgiye ihtiyaç duyulan ve geniş kitleler tarafindan kullanılan bir geliştirme kartıdır [7].

Türkiye geneli kodlama eğitimi üzerine tarama modeli ile yapılan bir çalışmada öğretmenlerin derslerde kullandıkları kodlama ve robotik setlerin başında \%55 oran ile Arduino ilk sırada gelmektedir. 27 çeşit setin yer aldığ 1 sıray $\% 11$ ile Makeblock mBot ve \%8 ile Makey Makey takip etmektedir. Çalışmada Scratch'ın \%88'lik oran ile en çok kullanılan platform olduğu belirtilmiştir [8]. Yine aynı çalışmada Arduino setleri, öğretmenler tarafindan; teknik yönden üstünlük, tüm platformlarca desteklenmesi, kolay temin edilebilmesi, kullanım kolaylığ 1 ve komutlara kisa sürede yanıt verebilmesi bakımından 5 farklı boyutta ilk sirada yer almaktadır. Kendilerine ait robotik ve kodlama seti bulunan öğrencilerin de \%62'si Arduino, \%14'ü Makeblock mBot ve $\% 10$ 'u ise Lego Mindstorm EV3 sahibidir [8].

Bilişim Teknolojileri öğretmenleri ile robotik kodlama üzerine yapılan diğer bir çalışmada ise öğretmenler, eğitsel robotların eğitimde kullanımının olumsuzlukları arasında; robotik setlerin maliyetlerinin fazla olması ve okulların donanımsal bakımdan eksiklerinin olması konularına değinmişlerdir [9].

Eğitsel robotik uygulamalar üzerine içerik analizi yöntemi ile 142 çalışmanın incelenerek yapıldığı başka bir çalışmada ise eğitsel robotik uygulamaların olumsuz yönlerinden bazıları; devre kurmakta zorlanma, uygun kod bloklarını bulamama, bazı kod bloklarının karışık olması, parça birleştirmenin zorluğu ve maliyetli olması şeklindedir [10].

mBlock yazılımı ise Makeblock robotları, Arduino, Littlebits vb dahil çeşitli robotları programlamak için yaygin kullanıma sahip blok tabanlı Scratch temelli bir kodlama platformudur [11]. Avantajlarından biri de uzantı (eklenti) yazlabilmesi ve canlı mod veya Scratch modu olarak adlandırılan modda yapılan kodlamaları Arduino geliştirme kartına yüklemeye gerek kalmaksızın [12] uygun aygıt yazılımı ile [13] anında çalıştırabilmesidir.

$\mathrm{Bu}$ çalşşmadaki amaç, temel kodlama ve robotik eğitimlerinde genellikle tercih edilen hazır robot kitlerine alternatif bir eğitim robotu geliştirmektir. Bu amaçla hem öğretmenler hem de öğrenciler tarafindan temin edilmesi ve montajı kolay; Arduino mikrodenetleyicisi ile çeşitli aktüatör ve sensörlere sahip düşük maliyetli bir eğitim robotu geliştirilmiştir. Ayrıca robotun blok tabanlı olarak programlanabilmesi için araştırmacı tarafindan bir mBlock uzantısı yazılmış ve yapılan programların Arduino'ya yüklemeye gerek kalmadan çalıştırılabilmesi için de robotun kendi aygit yazılımı oluşturulmuştur. Robot, yazılan uzantı ile mBlock v3 yazılımında kolayca programlanabilmekte; yapılan kodlamalan Scratch modunda USB ve bluetooth bağlantısı üzerinden eş zamanlı bir şekilde çalıştırabilmektedir. Robot temel kodlama ve robotik eğitimlerinde kullanılabilir ve istenirse amaca uygun farklı sensörler eklenerek geliştirilebilir.

\section{2. ÖNCEKİ ÇALIŞMALAR (PREVIOUS STUDIES)}

Literatürde çeşitli eğitim robotları ve kitleri bulunmaktadır. $\mathrm{Bu}$ nedenle konuya ilișkin bazı akademik çalışmalara yer verilmiştir.

Topuz, Çoban, Arslan ve Tufançlı (2019), elektronik ve robotik kodlama eğitimlerinde kullanılabilecek ekonomik ve işlevsel bir robotik eğitim seti geliştirmiştir. Robotun üzerinde Arduino geliştirme kartı ve eğitimlerde kullanılabilecek çeşitli elektronik devre elemanları ile modüller bulunmaktadır. Robotta Arduino geliştirme kartı kullanıldığından; Arduino IDE ile metin tabanlı olarak programlanabilmekte ve Scratch gibi blok mantığ ile programlama yapmaya izin veren yazilımlarla da uyumlu bir şekilde çalışabilmektedir [14].

Rodriguez ve Cuesta (2016), her öğrencinin ödevler, robotik projeler ve çeşitli görevleri gerçekleştirebilmesi için; ağa bağlı (TCP/IP, 3G, Wifi, Bluetooth) ve birçok sensöre sahip (kamera, GPS, ivme ölçer vb) düşük maliyetli mobil bir robot çalışması yapmıştır. Robot, sınıftaki öğrenciler ve uzaktan eğitim öğrencileri tarafindan kolayca programlanabilmekte ve yapılandırılabilmektedir [15].

Junior, Neto, Hernandez, Martins, Roger ve Guerra (2013), yeterli mali desteğe sahip olmayan okullar ve 
öğrencilerin erişebilmesini göz önünde bulundurarak düşük maliyetli Arduino Uno tabanl, öğrencilerin robotu kendisinin yapmasina olanak veren bir robotik kit tasarlamıştır. Robotta Arduino platformunun yanı sıra arayüz ve diş ortam ile iletişim için 2 elektronik kart daha bulunmaktadır. Ortaöğretim robotik atölyelerinde uygulanması amaçlanan robot, MiniBloq adında blok tabanlı programlama ortamında kullanılmıştır [16].

Filho, Almeida ve Martins (2011) okullarda robotik öğretiminde kullanılmak üzere elektronik atıklardan ve kolay elde edilebilir malzemelerden çok görevli ve altı tekerlekli mobil bir robot geliştirmiştir. Robotta PIC mikrodenetleyici, sensörler ve aktüatörler bulunmaktadır. Programın mikrodenetleyiciye kaydedilmesi doğrudan anakart üzerinden yapılabilmekte ve herhangi bir bileşen çıkarmaya gerek kalmamaktadır [17].

Lee, Kang, Lee, Lee ve Seo (2016), ilkokul öğrencilerine yönelik blok tabanlı olarak kontrol edilebilen bir eğitim robotu geliştirmiş ve ögrencilere uygulamıştır. Robot üzerinde çeşitli sensörler ve aktüatörler bulunmaktadır ve robot kendi uzantısı ile Scratch üzerinden kontrol edilebilmektedir. Robot, kamera ve renk izleme fonksiyonlarına ve gömülü MIC ve CDS ile dokunmatik, IR ve VR gibi çeşitli uygulamalar için çıkarılabilir sensörlere sahiptir. Arduino UNO ve 3. parti çeşitli sensörler ve aktüatörler ile uyumlu olan robot ve PC arasındaki iletişim bluetooth bağlantısı üzerinden sağlanmaktadır [18].

Topuz ve arkadaşları (2019) ile Lee ve arkadaşlarının (2016) çalışmalarının [14, 18] konuya en yakın çalışmalar olduğu görülmüştür. $\mathrm{Bu}$ çalışmada da temin edilmesi ve montajı kolay malzemeler ile geliştirilmeye açık, kendi mBlock uzantis1 ile programlanabilen, yapılan programları Arduino'ya yüklemeden mBlock Scratch modunda kendi aygıt yazılımı ile çalıştırabilen bir eğitim robotu geliş tirilmiştir.

\section{YÖNTEM (METHOD)}

$\mathrm{Bu}$ çalışmada robota, Arduino Nano geliştirme kartı kullanıldığı için myNanoBot adı verilmiştir. Robot ayrıca Arduino Uno ve Arduino Mega kartları ile de test edilmiştir. Testler mBlock v3.4.12 sürümünde gerçekleştirilmiştir. Robotun geliştirilme aşaması ise; temel çalışma prensibi, bluetooth modülü konfigürasyonu, gereken bileşenler ve montaj, robotun aygit yazılımı, robot seri port protokolü ve robotun mBlock uzantıs1 olarak 6 alt başlikta incelenmiştir.

\subsection{Temel Çalışma Prensibi (Basic Working Principle)}

mBlock platformunda Makeblock kontrol kartları ile birlikte Arduino geliştirme kartları da programlanabilmektedir [11]. mBlock programinda her blok, Scratch modu ve Arduino modu olmak üzere 2 moda sahiptir [12]. Scratch modunda robot veya Arduino kartının yapılan programın çalıştırılabilmesi için bilgisayara bağlı olması zorunda olup; bu modda seri port üzerinden haberleşme ile mBlock'taki bloklar robot veya Arduino ile birlikte etkileşimli olarak kullanılabilmektedir $[11,12]$. Scratch modu için robotta veya Arduino'da ilgili aygıt yazılımının yüklü olması gerekmektedir [11].

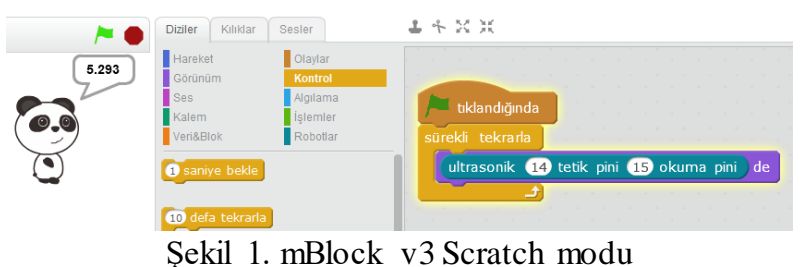

Şekil 1. mBlock v3 Scratch modu (mBlock v3 Scratch mode)

Arduino modunda ise yapilan programın robota veya Arduino'ya yüklenmesi gerekmekte olup bu modda robot veya Arduino, programı kendisi çalıştırabilmekte; ancak bilgisayar bağlantısı olmadığı için bu modda Scratch blokları kullanılamamaktadır [12]. Yapılan yükleme işleminden dolayı ise önceden yüklenmiş aygıt yazılımı silinmektedir [11].

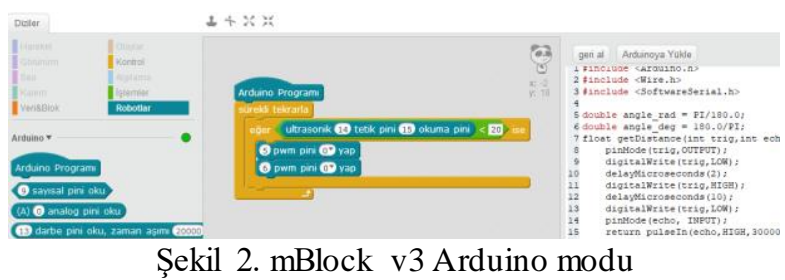

(mBlock v3 Arduino mode)

Scratch modunun kullanılabilmesi için robot veya geliştirme kartına mBlock programında "Bağlan" menüsünden "Aygıt Yazilımı Güncellemesi” seçeneği ile gerekli aygıt yazılımının yüklenmesi gerekmektedir [11]. Kullanılan robot veya geliştirme kartı türüne göre mBlock aygıt yazılımı yüklemesi yapmaktadır [11]. mBlock, mBot için mbot_firmware.ino [19] dosyasını, Arduino kartları için de orion_firmware.ino [20] dosyasını kaynak olarak göstermektedir. Aygit yazılımı yüklemesinin ardından; yapılan programlar mBlock tarafindan seri port haberleşmesi ile herhangi bir derleme/yükleme işlemine tabi tutulmadan anında çalıştırılmaktadır $[11,12]$.

Bu çalışmada da robotu Scratch modunda kullanabilmek için Arduino'ya robot için geliştirilen aygit yazılımı yüklenmiş ve robotu programlamayabilmek için de yazılan uzantı mBlock programına dahil edilmiştir.

\subsection{Bluetooth Modülü Konfigürasyonu (Bluetooth Module Configuration)}

Çalışmada bluetooth modülü olarak HC-06 kullanılmıştır. mBlock tarafindan mBot ile Arduino geliştirme kartları için kaynak gösterilen her 2 aygıt yazılımı [19, 20] incelendiğinde seri haberleşmenin 115200 baud rate değeri üzerinden yapıldığı görülmektedir. HC-06'nın ise varsayılan baud rate değeri 9600'dür [21]. Bu nedenle bluetooth modülünün baud rate değeri 115200 bps olarak değiştirilmiştir. Ayrıca etraftaki cihazlar ile karışıklığ 1 
önlemek amacıyla modülde isim ve şifre değişikliği yapılmıştır. Konfigürasyon için HC-06 modülü Arduino'ya bağlanarak tablo 1'deki AT komutları [21,22] Arduino IDE seri monitöründen modüle uygulanmıştır.

Tablo 1. HC-06'ya uygulanan AT komutları (AT commands applied to HC-06)

\begin{tabular}{|l|l|}
\hline \multicolumn{1}{|c|}{ Komut } & \multicolumn{1}{c|}{ Alınan Sonuç } \\
\hline AT+NAMEmyNanoBot & OKsetname \\
\hline AT+PIN5555 & OKsetPIN \\
\hline AT+BAUD8 & OK115200 \\
\hline
\end{tabular}

\subsection{Gereken Bileşenlerve Montaj (Required Components and} Assembly)

Robotta boyut faktörü ve analog pin kazancindan dolayı Arduino Nano ile avantajları sebebiyle sensörlerin ve aktüatörlerin de modülleri tercih edilmiştir. Örneğin LDR modülünden hem analog hem de dijital çıkışı ve hassasiyet ayarı ile ortam 1 şı̆ 1 ile ilgili veriler kolayca alınabilmektedir. Eğitim robotunda kullanılan bileşenlerin listesi tablo 2'de, robotun Arduino pin bağlantıları ise tablo 3'de sunulmuştur.

Tablo 2. Robot için gerekli bileșenler (Required components for the robot)

\begin{tabular}{|l|c|}
\hline \multicolumn{1}{|c|}{ Bileşen } & Adet \\
\hline Şasi & 1 \\
\hline Teker & 2 \\
\hline DC motor(6V) & 2 \\
\hline Sarhoş teker & 1 \\
\hline Alt1lı (dörtlü+ikili) pil yuvas1 & 1 \\
\hline Anahtar & 1 \\
\hline Arduino Nano & 1 \\
\hline Motor sürücü kartı (L298N) & 1 \\
\hline Bluetooth modülü (HC-06) & 1 \\
\hline Mesafe sensörü (HC-SR04) & 1 \\
\hline RGB LED modülü & 1 \\
\hline Iş1k sensörü modülü & 1 \\
\hline Ses sensörü modülü & 2 \\
\hline Buzzer modülü & 1 \\
\hline Mini breadboard & 6 \\
\hline Mesafe sensörü tutucu aparatı & \\
\hline AA pil & 1 \\
\hline
\end{tabular}

Tablo 3. Robotun Arduino pin bağlantıları (Arduino pin connections of the robot)

\begin{tabular}{|l|c|l|c|}
\hline \multicolumn{1}{|c|}{ Devre Elemanı } & Pin & \multicolumn{1}{c|}{ Devre Elemanı } & Pin \\
\hline Bluetooth TX & RX0 & RGB Kırmız1 & D9 \\
\hline Bluetooth RX & TX1 & RGB Yeşil & D10 \\
\hline L298N IN1 & D2 & RGB Mavi & D11 \\
\hline L298N IN2 & D3 & Buzzer Sinyal & D12 \\
\hline LDR Dijital & D4 & HC-SR04 Trig & A0 \\
\hline L298N ENA & D5 & HC-SR04 Echo & A1 \\
\hline L298N ENB & D6 & LDR Analog & A2 \\
\hline L298N IN3 & D7 & Ses Dijital & A3 \\
\hline L298N IN4 & D8 & Ses Analog & A4 \\
\hline
\end{tabular}




\subsection{Robot Seri Port Protokolü (The Robot Serial Port Protocol)}

Makeblock mBot seri port protokolünde Makeblock kontrol kartlarıyla uygulamaların nasıl haberleşeceği detaylı bir şekilde açıklanmış olup geliştirilen robot için yapılan uyarlama sonucu mikrodenetleyiciye gönderilen çerçeve formatı [13] şu şekilde olmuştur:

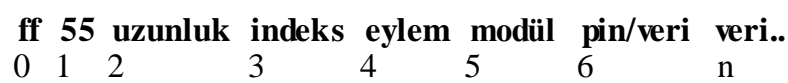

Tablo 4.Mikrodenetleyiciye gönderilen çerçeve yapıs1[13] (Instruction frame format sent to the microcontroller [13])

\begin{tabular}{|c|l|l|}
\hline Bayt & İsim & Açıklama \\
\hline 0 & Ön ek 1 & 0xff \\
\hline 1 & Ön ek 2 & $0 x 55$ \\
\hline 2 & Uzunluk & Bu bayttan sonra kaç bayt gönderildiği \\
\hline 3 & İndeks & Yanıtta gönderilecek takip numaras1 \\
\hline 4 & Eylem & Sensör için 0x01, aktüatör için 0x02 \\
\hline 5 & Modül & Aygit yazılımındaki sensör/aktüatör kodu \\
\hline 6 & Pin/Veri & Pin numaras1 vey a veri \\
\hline $\mathrm{n}$ & Veri & Veri \\
\hline
\end{tabular}

Örneğin robottaki mesafe sensöründen uzaklık talebinde mikrodenetleyiciye aşağıdaki çerçeve gönderilmektedir:

\section{ff $\begin{array}{lllll}55 & 03 & 00 & 01 & 01\end{array}$}

İlgili veride ilk 2 bayt (ff ve 55) ön ektir. Üçüncü bayt (03) bu bayttan sonraki verinin uzunluğunu (3 bayt gönderildiğini) bildirmektedir. Dördüncü bayt (00) indeks (takip) numarasıdır. Beşinci bayt (01) bir sensörden veri talep edildiğini belirtir. Altıncı bayt (01) ise bu sensörün kod numarasıdır ve karşılı̆ğ aygıt yazılımında tanımlanmış “ULTRASONIC_SENSOR”dür.

Mikrodenetleyiciye gönderilen çerçeve, aygıt yazılımı tarafindan ayrıştırıldıktan sonra işlemin sonucu gerektiğinde yine belirli bir formatta geri gönderilmektedir. Makeblock mBot seri port protokolünde sensörlerden veri talep edildiğinde mikrodenetleyicinin yanıt formatı [13] ise şu şekildedir:

\section{ff 55 indeks veri türü veri 0d 0a.

$\begin{array}{lllllll}0 & 1 & 2 & 3 & \ldots & \mathrm{n} & \mathrm{n}-1\end{array}$

Tablo 5. Mikrodenetleyiciden gelen yanıt formatı [13] (Response data format from the microcontroller [13])

\begin{tabular}{|l|l|l|}
\hline Bayt & İsim & Açıklama \\
\hline 0 & Ön ek 1 & 0xff \\
\hline 1 & Ön ek 2 & 0x55 \\
\hline 2 & İndeks & Talep indeks numaras1 \\
\hline 3 & Veri türü & Gönderilen verinin türü \\
\hline$\ldots$ & Veri & Gönderilen veri \\
\hline $\mathrm{n}-1$ & Son ek 1 & 0x0d \\
\hline $\mathrm{n}$ & Son ek 2 & 0x0a \\
\hline
\end{tabular}

Örneğin robottaki mesafe sensöründen uzaklı talep edildiğinde mikrodenetleyicinin gönderdiği yanıt aşağıdakine benzer olmaktadır [13]:

\section{ff $\begin{array}{lllllllll}55 & 00 & 02 & 00 & 00 & 88 & 43 & 0 d & 0 a\end{array}$}

İlk 2 bayt (ff ve 55) ön ek, son 2 bayt (0d ve 0a) ise son ektir. Üçüncü bayt (00) talep komutunda gönderilen indeks numarasıdır. Dördüncü bayt (02) gönderilen verinin tipinin float olduğunu belirtmekte olup ortada kalan diğer 4 bayt ise ölçülen uzaklık değeridir ve uygun dönüşümile kullanılabilir [13].

\subsection{Robotun mBlock Uzantisl (mBlock Extension of the Robot)}

mBlock uzantı rehberinde bir mBlock uzantısının nasıl yazılacağı detaylı bir şekilde açıklanmıştır. Buna göre her mBlock uzantıs1, basitçe; bir .js uzantılı ve bir de .s2e uzantılı iki dosya içeren sıkıştırılmış bir klasördür [12]. Kılavuz çerçevesinde uzantı için .s2e ve .js dosyaları oluşturularak robot kontrolü için blok tanımlamaları uyarlanan seri port protokolü çerçevesinde yapılmıştır. .s2e ile js dosyasından örnek birer blok tanımı ve ilgili Javascript fonksiyonu kodları aşağıda sunulmuştur.

\subsection{1. s2e Dosyast (s2e File)}

.s2e dosyas1, uzant1 ile ilgili temel bilgilerin ve blok tanımlamalarının yapıldığ 1 , tanımlanan blokların Arduino modunda üreteceği Arduino kodlarının ve blok çevirilerinin yer aldığı JSON formatında "Scratch 2 uzantıs1" temelinde bir dosyadır [12]. Robottaki RGB LED'i istenen değerlere göre yakabilen mBlock uzantısında tanımlanan blok şekil 5'te örnek olarak gösterilmektedir.

\section{RGB Ledi Kırmızı $0 \gg$ Yeşil $0 \gg$ Mavi $0 \gg$ değerinde yak}

Şekil 5. RGB LED bloğu (RGB LED block)

Bu bloğu oluşturmak için .s2e dosyasında yapılan blok tanımlama kodu ise aşağıdaki gibidir:

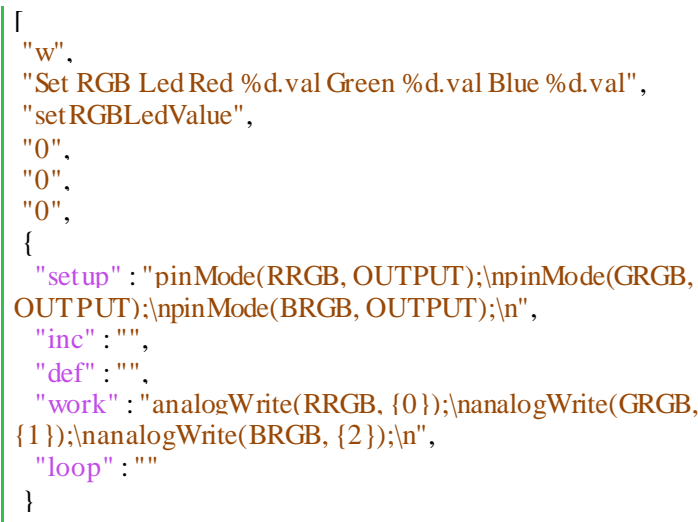

Şekil 6. RGB LED blok tanımlama kodu (RGB LED block definition code) 
RGB bloğunu oluşturan satırlara ilişkin açıklamalar tablo 6'da gösterilmektedir:

Tablo 6. RGB LED blok tanımlama satırları [12] (RGB LED block definition lines [12])

\begin{tabular}{|c|l|}
\hline Satır & Açıklama \\
\hline 2 & Blok tipi (w: aktüatör çalıştırma) \\
\hline 3 & Blok metni ve parametreleri \\
\hline 4 & .js dosyasında çalıştırılacak fonksiyon \\
\hline $5,6,7$ & Blok parametreleri varsayılan değerleri \\
\hline 9 & Arduino void setup \{\} için üretilecek kod \\
\hline 11 & Arduino'da eklenecek kütüphaneler \\
\hline 12 & Arduino'da tanımlanacak değişkenler \\
\hline 13 & Arduino'da blok için üretilecek kod \\
\hline 15 & Arduino void loop \{\} için üretilecek kod \\
\hline
\end{tabular}

mBlock uzantı rehberi dikkate alınarak robotu programlayabilmek için yazılan uzantıda tanımlanan bütün bloklar şekil 7'de gösterilmektedir. Bloklar robotu kontrol edecek şekilde ve kafa karıșıklığını önlemek amaciyla mümkün olduğunca sade bir şekilde oluşturulmaya çalışılmıştır.

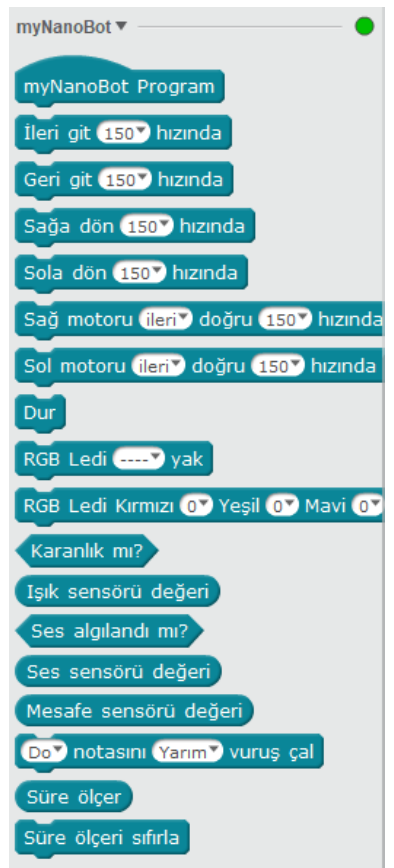

Şekil 7. Robotun mBlock uzantısı (mBlock extension for the robot)

\subsection{2.js Dosyasl (js File)}

.js dosyas 1 ise .s 2e dosyasinda tanımlanan uzantıya ait blokların; robot veya Arduino ile mBlock arasinda Scratch modunda etkileşimli bir şekilde çalışabilmesi için gerekli fonksiyonların tanımlandığ 1 ve seri haberleşme ile ilgili kodlamaların yapıldığ

mBlock Arduino uzantısı arduino.js [23] incelendiğinde protokol çerçevesinde seri haberleşme ile ilgili kodlamaların yapıldığ 1 , ilgili kod bloklarına ait fonksiyonların da oluşturulduğu görülmektedir. Buna göre veriler sendPackage fonksiyonu aracıllı̆ı ile gönderilmekte olup bir sensörden veri talep etmek için getPackage, bir aktüatör için de runPackage fonksiyonu ön plana çıkmaktadır:

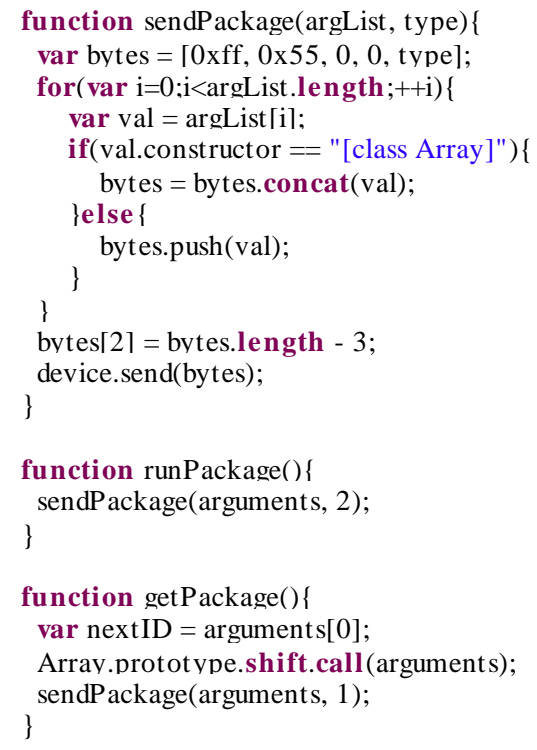

Şekil 8. arduino.js çerçeve gönderme fonksiyonları [23] (arduino.js frame sending functions [23])

Mikrodenetleyiciye çerçeve gönderirken; gönderilmesi gereken ön ek, uzunluk ve indeks verisi ile eylem (sensör/aktüatör) kodunun protokole uygun sirayla "bytes" dizisine yerleştirildiği, diğer argümanların ise getPackage/runPackage tarafindan beklendiği görülmektedir. mBlock Arduino uzantıs1 .js dosyası [23] ve mBlock demo uzantısı [24] baz alınarak robot kontrolü için .s2e dosyasında tanımlanan bloklara ait Javascript fonksiyonları oluşturulmuştur. Geliştirilen robotta bulunan RGB LED'i istenen değerlere göre yakabilen Javascript fonksiyonu örnek olarak aşağıda sunulmuştur:

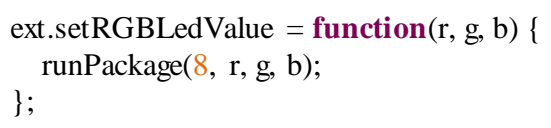

Şekil 9. RGB LED Javascript fonksiyonu (RGB LED Javascript function)

setRGBLedValue, .s2e dosyasında kod bloğu tanımlaması yapılırken belirlenmiş çalıştırılacak Javascript fonksiyonudur. $r, g$ ve $b$ parametreleri ise blokta tanımlanan ve Scratch modunda kullanıcıdan gelecek değerleri alacak değişkenlerdir. 8 değeri ise aygit yazılımındaki "RGBLED" sabitine karşıllı gelmekte ve RGB LED'i yakabilmek için aygıt yazılımındaki ilgili fonksiyona argüman olarak gönderilmektedir. Görüldüğü gibi RGB LED'i yakabilmek için aygıt yazılımında ihtiyaç duyulan argümanlar olan; aktüatör kodu (8=RGBLED) ile $\mathrm{r}, \mathrm{g}$ ve $\mathrm{b}$ değerleri runPackage fonksiyonu vasıtası ile mikrodenetleyiciye gönderilmiş olmaktadır. 


\section{BULGULAR (FINDINGS)}

$\mathrm{Bu}$ aşamada öncelikle robot için geliştirilen aygıt yazılım Arduino geliştirme kartına yüklenmiştir. $\mathrm{Bu}$ işlem için Arduino, USB kablo ile bilgisayara bağlanıp Arduino IDE programında uygun kart türü ve port seçimi yapılmıştır. TX ve RX pin bağlantıları yükleme esnasından önce çıkarılmıştır. Aygıt yazılımı karta yüklendikten sonra TX ve RX pin bağlantıları tekrar yapılmıştır.

Aygıt yazılımı yüklemesinin ardından robot için yazılan uzant 1 mBlock programına dahil edilmiştir. Uzantının eklenmesinin ardından işletim sisteminde bluetooth modülü ile bilgisayar eşleşmesi yapılmıştır. Robot ile mBlock arasında bluetooth üzerinden bağlantı kurularak Scratch modunda uzantıya ait blokların çift tıklanarak çalışıp çalışmadığı test edilmiştir.

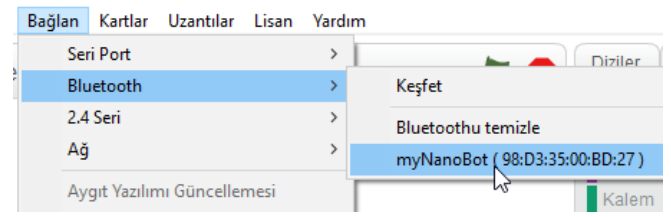

Şekil 10. Robot ile bluetooth bağlantısı kurulması

(Establishing a bluetooth connection with the robot)

Robotun sahip olduğu her sensör ve aktüatör, hem Scratch modu hem Arduino modu için farklı uygulamalar ile Arduino Nano, Uno ve Mega geliştirme kartları kullanılarak ayrı ayrı test edilmiştir. Scratch modunda yoğun seri port trafiği oluşturacak uygulamalar ile çeşitli denemeler yapılmıştır. Scratch ve Arduino modu için örnek 2 uygulama aşağıda sunulmuştur.

\subsection{Scratch Modu Örnek Uygulama (Example Program for Scratch Mode)}

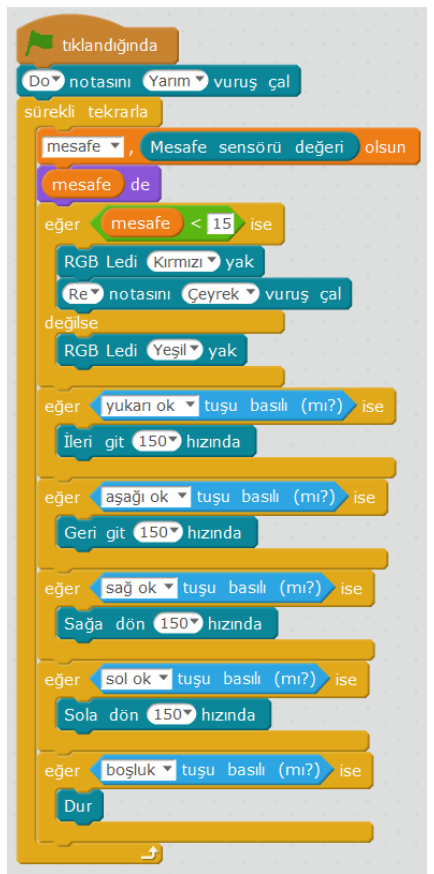

Şekil 11. Scratch modu örnek uygulama (Example program for Scratch mode)
Scratch modunda robotun bazı temel özellikleri şekil 11'deki uygulama ile test edilmiștir. Uygulama başlayınca "do" notası çalmaktadır. Yön tuşları ile robotun ileri, geri, sağ ve sol yönlerinde kontrolü sağlanabilmekte; boşluk tuşuna basılınca ise robot durmaktadır. Bununla birlikte sürekli mesafe ölçümü yapılmakta ve robot $15 \mathrm{~cm}$ altında herhangi bir engel ile karşılaştığında RGB LED kırmızı yanmakta ve "re" notası ile uyarı vermektedir. Mesafe 15 $\mathrm{cm}$ ve üzerinde ise RGB LED yeşil yanmaktadır.

Arduino Nano, Uno ve Mega kartları ile test edilen uygulama yoğun seri port trafiği oluşturan uygulamalardan biridir. Sürekli mesafe ölçümü yapılmakta ve ayrıca birden fazla yön tuşuna aynı anda bas 1labilmektedir.

Her 3 geliştirme kartı ile test edilen uygulamada herhangi bir problem ile karşıllaşılmamıştır.

\subsection{Arduino Modu Örnek Uygulama (Example Program for Arduino Mode)}

Robot, Arduino modu için şekil 12'deki uygulama ile test edilmiştir.

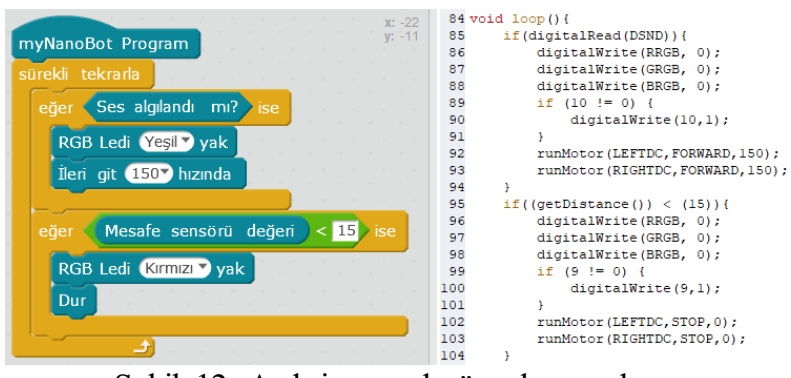

Şekil 12. Arduino modu örnek uygulama (Example program for Arduino mode)

Uygulamada robot, ses algıladığında ileri doğru hareket etmekte ve RGB LED yeşil yanmaktadır. Sürekli mesafe ölçümü yapılmakta ve mesafe 15 cm'nin altına düştüğünde ise robot durarak RGB LED kırmızı yanmaktadır. Arduino modu için otomatik oluşturulan kodlar şekil 12'nin sağ kısmında yer almaktadır.

Örnek uygulamayı robota yüklemek için USB kablo ile bağlantı sağlanmış; TX ve RX pin bağlantıları kesildikten sonra yükleme işlemi yapılmıştır. Arduino Uno, Mega ve eski önyükleyiciye sahip Arduino Nano geliştirme kartlarında mBlock tarafindan yapılan yükleme işleminde herhangi bir problem yaşanmaz iken güncel önyükleyiciye sahip Arduino Nano kartlarda yükleme işlemi başarısız olmuştur. Güncel önyükleyiciye sahip Arduino Nano kartlarda programın yükleme işlemi Arduino IDE üzerinden Arduino modu için üretilen kodların kopyalanması ile gerçekleştirilmiştir.

Arduino geliştirme kartlarına yüklenen uygulama bilgisayar bağlantısı olmadan çalışmış ve her 3 geliştirme kartında da herhangi bir sorun ile karşıllaşılmamış tır. 


\section{SONUÇ (CONCLUSION)}

$\mathrm{Bu}$ çalışmada geliştirilen robot, kodlama ve robotik eğitimlerinde kullanılmak için tasarlanmış Makeblock $\mathrm{mBot}$ benzeri model bir robottur. mBot ve diğer türevleri gibi Arduino tabanlidır. Temel, genel ve uygun fiyath robotik malzemeler kullanılarak montajı yapılmıştır. Arduino Nano yerine Arduino Uno ve Arduino Mega kullanılması da mümkündür. mBot'un başlica temel özelliklerine sahiptir ve geliştirilmeye açıktır. Yazılan uzantı ile birlikte kodlama ve robotik eğitimleri başlangıç seviyesinde rahatlkla kullanılabilir. Uzantıdaki bloklar öğrencilerin kafa karışıklığını önlemek amacıyla mümkün olduğunca sadeleştirilmiş̧tir.

Yazlan uzant1, mBlock (3. sürüm) Scratch ve Arduino modunu desteklemektedir. Scratch modunda yapilan kodlamalarn neticesi herhangi bir derleme/yükleme işlemine tabi tutulmadan hem USB hem de bluetooth bağlantısı üzerinden görülebilmekte; istendiğinde yapılan uygulama USB kablo bağlantısı ile robota kalıcı olarak da yüklenebilmektedir.

Scratch modunun kullanılabilmesi için geliştirilen aygıt yazilımının robota yüklenmesi gerekmektedir. Scratch modunda derleme ve yükleme işlemine gerek olmaması hem zaman kazandırmakta hem de öğrenmeyi pratikleştirmektedir. $\mathrm{Bu}$ modda mBlock ile robottaki sensör ve aktüatörlerin etkileşimli kullanılabilmesi ise kodlama becerilerinin kazanılmasını kolaylaştırmaktadır. Bununla birlikte Scratch modunda robot, Arduino moduna göre; seri port haberleşmesinden dolayı isteklere anında tepki veremeyebilmektedir.

Robotu hareket ettirmek için ilk esnada DC motorlar fazla akım çekebilmekte; piller robottaki diğer elektronik malzemelere gereken gerilimi/akımı sağlayamadığı durumda ise bluetooth bağlantısı kesilebilmektedir. Bu nedenle uygulamada yüksek akım verebilen piller tercih edilmelidir. Ayrıca bu çalş̧mada eğitim robotu geliştirilirken öğretmenler ve öğrenciler tarafindan temin edilmesi ve montajı kolay, düşük maliyetli bileşenler tercih edilmiştir. Bununla beraber eğitim robotu tümleşik devre yapısı kullanılarak da geliştirilebilir. Böylelikle hem karşılaşılabilecek problemler en aza indirilebilir hem de robotun boyutları küçültülebilir.

Robot geliştirilmeye açı bir robottur. $\mathrm{Bu}$ nedenle çalş̧mada hem Scratch modu hem de Arduino modu için yazılan mBlock uzantısina ve robot için uyarlanan Makeblock mBot seri port protokolü ile bu haberleşmede kullanılan çift yönlü çerçeve yapısına robot üzerinden örneklerle yer verilmiştir. Robot uyarlanan protokole göre geliştirilebilecek tablet, akıllı telefon veya PC uygulamaları ile kontrol edilebilir ve ayrica blok tabanlı programlama yapabilen farklı yazlımlara uygun değişikliklerle entegre edilebilir.

\section{KAYNAKLAR (REFERENCES)}

[1] İnternet: CodingBK, Geleceğin Dili Kodlama, https://www.bahcesehir.k12.tr/tr/images/pdf/codingbksayi1.pdf, 08.01.2021.

[2] Ş. Sayginer, Blok Tabanlı Görsel ve Metin Tabanlı Programlama Öğretimlerinin Erişi, Mantıksal Düşünme ve Motivasyona Etkileri, Yüksek Lisans Tezi, Hacettepe Üniversitesi, Eğitim Bilimleri Enstitüsü, 2017.

[3] R. Kraleva, V. Kralev, D. Kostadinova, "A Methodology for the Analysis of Block-Based Programming Languages Appropriate for Children", Journal of Computing Science and Engineering, 13(1), 1-10, 2019.

[4] J. Maloney, M. Resnick, N. Rusk, B. Silverman, E. Eastmond, "The Scratch Programming Language and Environment", ACM Transactions on Computing Education, 10(4), 16, 2010.

[5] T. Talan, "Investigation of the Studies on the Use of Scratch Soft ware in Education", Journal of Education and Future, 18, 95 111,2020

[6] İnternet: Y. Tekin, Kodlama ve Robotik Eğitimi, https://www.aknetakademi.com.tr/blog-yazilari-vemakaleler/kodlama-ve-robotik-egitimi, 05.01.2020.

[7] O. Duman, "Eğitimde Arduino Kullanımı ile İlgili Yapılan Çalışmalar”, XII. Ulu slararası Eğitim Araştırmaları Kongresi, Rize, 489-499, 2019.

[8] M. Sayın, Kodl ama ve Robotik Eğitim Setlerinin Öğretmen ve Öğrenci Gözüyle Değerlendirilmesi, T.C. MEB Yenilik ve Eğitim Teknolojileri Genel Müdürlüğü, 2019.

[9] F. N. Aksu, Bilişim Teknolojileri Öğretmenleri Gözünden Robotik Kodlama ve Robotik Yarışmaları, Yüksek Lisans Tezi, Balıkesir Üniversitesi, Fen Bilimleri Enstitüsü, 2019.

[10] T. Talan, "Eğitsel Robotik Uygulamaları Üzerine Yapılan Çalışmaların İncelenmesi”, Yaşadıkça Eğitim, 34 (2), 503-522, 2020 .

[11] İnternet: $\mathrm{W} . \mathrm{Yu}$, Getting Started with mBlock, http://download.makeblock.com/mblock/docs/getting-startedwith-mblock.pdf, 25.12.2019.

[12] İnternet: W. Yu, Creating mBlock Extensions, http://download.makeblock.com/mblock/mblock_extension_guide .pdf, 15.02.2020.

[13] İnternet: Makeblock mBot Protocol,

https://www.makeblock.com/project/makeblock-orion-protocol, 03.04.2020.

[14] A. C. Topuz, H. H. Çoban, S. Arslan, S. Tufançlı, "Ekonomik ve İşlevsel Bir Robotik Eğitim Setinin Geliştirilmesi: ARUbot", Ahmet Keleşoğlu Eğitim Fakültesi Dergisi, 1 (2), 121-138, 2019.

[15] F. M. L. Rodriguez, F. Cuesta, “Andruino-A1: Low-Cost Educational Mobile Robot Based on Android and Arduino", Journal of Intelligent \& Robotic Systems, 81(1), 63-76, 2016.

[16] L. Junior, O. T. Neto, M. F. Hernandez, P. S. Martins, L L. Roger, F. A. Guerra, "A Low-Cost and Simple Arduino-Based Educational Robotics Kit”, Multidisciplinary Journals in Science and Technology, Journal of Selected Areas in Robotics and Control (JSRC), 3 (12) 1-7, 2013. 
[17] J. A. B. L. Filho, W. R. M. Almeida, S. G. Martins, "Development of a mult itasking mobile robot for the construction of educational robotics kits", 2011 International Conference on ElectronicDevices, Systems and Applications (ICEDSA), Malaysia, 213-216, 2011

[18] Y. D. Lee, J. J. Kang, K. Y. Lee, J. Lee, Y. Seo, “The Development of an Educational Robot and Scratch-based Programming", International Journal of Advanced Smart Convergence, 5(2), 8$17,2016$.

[19] İnternet: mbot_firmware.ino, https://github.com/Makeblockofficial/Makeblock-

Libraies/blob/master/examples/Firmware_For_mBlock/mbot_fir mware/mbot_firmware.ino, 20.02.2020
[20] İnternet: orion firmware.ino, https://github.com/Makeblockofficial/Makeblock-

Libraies/blob/master/examples/Firmware_For_mBlock/orion_fir mware/orion_firmware.ino, 20.02.2020.

[21] Internet: Guangzhou HC Information Technology Co., Ltd. Product Data

Sheet, https://www.olimex.com/Products/Components/RF/BLUETOOT H-SERIAL-HC-06/resources/hc06.pdf, 05.03.2020.

[22] Internet: M. Currey, HC-06 (ZG-B23090W) Bluetooth 2.0 EDR modules, http://www.martyncurrey.com/hc-06-zg-b23090wbluetooth-2-0-edr-modules/, 11.03.2020.

[23] İnternet: arduino.js, https://github.com/Makeblockofficial/mBlock/blob/master/src/ext /libraries/arduino/js/arduino.js, 15.04.2020.

[24] İnternet: Wangyu, A Demo Extension for Arduino, https://www.mblock.cc/extensions/uploads/792fcf56085fc19ed4d f4d260fb9fb87483ca2c2.zip, 07.04.2020. 